\title{
ON THE CONVERGENCE OF A LINEAR TWO-STEP FINITE ELEMENT METHOD FOR THE NONLINEAR SCHRÖDINGER EQUATION*
}

\author{
Georgios E. Zouraris ${ }^{1}$
}

\begin{abstract}
We discretize the nonlinear Schrödinger equation, with Dirichlet boundary conditions, by a linearly implicit two-step finite element method which conserves the $L^{2}$ norm. We prove optimal order a priori error estimates in the $L^{2}$ and $H^{1}$ norms, under mild mesh conditions for two and three space dimensions.
\end{abstract}

Mathematics Subject Classification. 65M12, 65M60.

Received: January 14, 2000.

\section{INTRODUCTION}

\subsection{The i.b.v. problem}

Let $d=1,2$ or 3 , and $\Omega \subset \mathbb{R}^{d}$ be a bounded domain with smooth boundary $\partial \Omega$. For $t^{*}>0$, we set $I_{*}:=\left[0, t^{*}\right]$ and consider the following initial and boundary value problem for the nonlinear Schrödinger equation:

$$
\begin{array}{ll}
w_{t}=\mathrm{i} \Delta w+\mathrm{i} f\left(|w|^{2}\right) w & \text { in } \Omega \times I_{*}, \\
w=0 & \text { on } \partial \Omega \times I_{*}, \\
w(x, 0)=w^{0}(x) & \text { for } x \in \Omega,
\end{array}
$$

where $w^{0}: \bar{\Omega} \longrightarrow \mathbb{C}$ and $f \in C^{3}([0,+\infty) ; \mathbb{R})$ are given functions. The nonlinear Schrödinger equation, often, appears as a model in mathematical physics (see $[1,7,8,16,22,26]$ ); for more information on the theory and applications we refer to $[5,6,10,14,15,17,19,21,25]$ and the references therein. In the sequel, we will assume that problem (1.1) admits a unique solution which is sufficiently smooth for our purposes.

\subsection{Notation and preliminaries}

For integer $s \in \mathbb{N}_{0}$, we denote by $H^{s}(\Omega)$ the Sobolev space consisting of complex-valued functions which, along with their distributional derivatives of order up to $s$, are in $L^{2}(\Omega)$, and by $\|\cdot\|_{s}$ the corresponding norm. The inner product on $L^{2}(\Omega)=H^{0}(\Omega)$ is denoted by $(\cdot, \cdot)$ and the associated norm by $\|\cdot\|$. $H_{0}^{1}(\Omega)$ consists of

Keywords and phrases. Nonlinear Schrödinger equation, two-step time discretization, linearly implicit method, finite element method, $L^{2}$ and $H^{1}$ error estimates, optimal order of convergence.

* Work supported by the EU-TMR project HCL \# ERBFMRXCT960033 and the EU-HCM scheme Reaction Diffusion Equations \# ERBCHRXCT930409.

1 Department of Numerical Analysis and Computing Science (NADA), Royal Institute of Technology (KTH), 10044 Stockholm, Sweden.

Present address: Centre de Recherche en Mathématiques de la Décision (CEREMADE), UMR CNRS 7534, Université de Paris IX-Dauphine, Place du Maréchal de Lattre-de-Tassigny, 75775 Paris Cedex 16, France. 
the functions of $H^{1}(\Omega)$ that vanish at $\partial \Omega$ in the sense of trace. In addition, for $m \in \mathbb{N}$ and for $u, v \in\left(L^{2}(\Omega)\right)^{m}$ we use $(u, v):=\sum_{j=1}^{m}\left(u_{j}, v_{j}\right)$ and $\|u\|:=\{(u, u)\}^{1 / 2}$. Finally, we set $\mathcal{H}:=H_{0}^{1}(\Omega) \cap C(\bar{\Omega})$, and denote by $|\cdot|_{p}$ the standard norm on $L^{p}(\Omega)$ for $p \geq 2\left(\right.$ i.e. $\left.|\cdot|_{2}=\|\cdot\|\right)$.

We mention the Poincaré-Friedrichs inequality

$$
\|v\| \leq \mathcal{C}_{\Omega}\|\nabla v\|, \quad \forall v \in H_{0}^{1}(\Omega),
$$

and the Sobolev-type inequalities

$$
|v|_{\infty} \leq \mathcal{C}_{1, \infty}\|\nabla v\|^{\frac{1}{2}}\|v\|^{\frac{1}{2}}, \quad \forall v \in H_{0}^{1}(\Omega), \quad d=1,
$$

and

$$
|v|_{s} \leq \mathcal{C}_{2, s}\|\nabla v\|^{\frac{s-2}{s}}\|v\|^{\frac{2}{s}}, \quad \forall s \in[2,+\infty), \quad \forall v \in H_{0}^{1}(\Omega), \quad d=2 .
$$

For an integer $r \geq 2$, let $\left\{S_{h}\right\}_{h \in(0,1)}$ be a family of finite dimensional subspaces of $\mathcal{H}$ satisfying

$$
\inf _{\chi \in S_{h}}\left\{\|v-\chi\|+h\|v-\chi\|_{1}\right\} \leq C h^{s}\|v\|_{s}, \quad \forall v \in H^{s}(\Omega) \cap H_{0}^{1}(\Omega), \quad s=2, \ldots, r, \quad \forall h \in(0,1) .
$$

For $h \in(0,1)$, we define the discrete Laplacian $\Delta_{h}: S_{h} \longrightarrow S_{h}$ by

$$
\left(\Delta_{h} \varphi, \chi\right)=-(\nabla \varphi, \nabla \chi), \quad \forall \varphi, \chi \in S_{h},
$$

an elliptic projection operator $R_{h}: H^{1}(\Omega) \longrightarrow S_{h}$ by

$$
\left(\nabla R_{h} v, \nabla \chi\right)=(\nabla v, \nabla \chi), \quad \forall v \in H^{1}(\Omega), \quad \forall \chi \in S_{h},
$$

and finally $P_{h}$ will be the $L^{2}$-projection operator onto $S_{h}$. The elliptic projection $R_{h}$ has the following approximation property $(c f .$, e.g., $[23])$

$$
\left\|R_{h} v-v\right\|+h\left\|R_{h} v-v\right\|_{1} \leq C_{R} h^{s}\|v\|_{s}, \quad \forall v \in H^{s}(\Omega) \cap H_{0}^{1}(\Omega), \quad s=2, \ldots, r, \quad \forall h \in(0,1),
$$

and obviously satisfies

$$
\left\|\nabla R_{h} v\right\| \leq\|\nabla v\|, \quad \forall v \in H^{1}(\Omega), \quad \forall h \in(0,1) .
$$

We will say that $f$ has the property $(\mathcal{D})$, if there exists $\varrho \geq 1$ such that

$$
\left|f^{\prime}\left(x^{2}\right) x\right| \leq C_{D}\left(1+x^{\varrho-1}\right), \quad \forall x>0 .
$$

\subsection{The numerical method}

Let $h \in(0,1), N \in \mathbb{N}, k:=\frac{t^{*}}{N}, t^{n}:=n k$ for $n=0, \ldots, N$, and $t^{1 / 2}:=t^{0}+\frac{k}{2}$. Let, also, $W_{h}^{0} \in S_{h}$ be a given approximation of $w^{0}$. First, we construct an approximation $W_{h}^{1 / 2} \in S_{h}$ of $w\left(\cdot, t^{1 / 2}\right)$ by

$$
\frac{W_{h}^{1 / 2}-W_{h}^{0}}{(k / 2)}=\mathrm{i} \Delta_{h}\left(\frac{W_{h}^{1 / 2}+W_{h}^{0}}{2}\right)+\mathrm{i} P_{h}\left[f\left(\left|W_{h}^{0}\right|^{2}\right)\left(\frac{W_{h}^{1 / 2}+W_{h}^{0}}{2}\right)\right] .
$$

Then, for $m=1, \ldots, N$, we define an approximation $W_{h}^{m} \in S_{h}$ to $w\left(\cdot, t^{m}\right)$ recursively by

$$
\frac{W_{h}^{1}-W_{h}^{0}}{k}=\mathrm{i} \Delta_{h}\left(\frac{W_{h}^{1}+W_{h}^{0}}{2}\right)+\mathrm{i} P_{h}\left[f\left(\left|W_{h}^{1 / 2}\right|^{2}\right)\left(\frac{W_{h}^{1}+W_{h}^{0}}{2}\right)\right]
$$


and

$$
\frac{W_{h}^{n}-W_{h}^{n-2}}{2 k}=\mathrm{i} \Delta_{h}\left(\frac{W_{h}^{n}+W_{h}^{n-2}}{2}\right)+\mathrm{i} P_{h}\left[f\left(\left|W_{h}^{n-1}\right|^{2}\right)\left(\frac{W_{h}^{n}+W_{h}^{n-2}}{2}\right)\right], \quad n=2, \ldots, N .
$$

Remark 1.1. By dropping (1.8a) and setting $W_{h}^{1 / 2}=W_{h}^{0}$ in (1.8b), an alternative method is obtained. For this method, our analysis also applies, but $W_{h}^{1}$ is a suboptimal order approximation of $w\left(\cdot, t^{1}\right)$ in the $H^{1}$ norm. This is the reason that the "fractional" step (1.8a) has been introduced.

Remark 1.2. Let $h \in(0,1)$. For given $\varphi \in S_{h}$ and $\lambda>0$, we define $T_{h}(\lambda, \varphi ; \cdot): S_{h} \longrightarrow S_{h}$ by

$$
T_{h}(\lambda, \varphi ; \chi):=\chi-\mathrm{i} k \lambda \Delta_{h} \chi-\mathrm{i} \lambda k P_{h}\left(f\left(|\varphi|^{2}\right) \chi\right) \text { for } \chi \in S_{h}
$$

To ensure that the method (1.8) is well-defined (i.e. the existence and uniqueness of $W_{h}^{1 / 2}$ and $\left\{W_{h}^{n}\right\}_{n=1}^{N}$ ), it is enough to show that $T_{h}(\lambda, \phi ; \cdot)$ is invertible. Let $\psi \in S_{h}$ be such that $T_{h}(\lambda, \varphi ; \psi)=0$. Then, we have $\operatorname{Re}\left(T_{h}(\lambda, \varphi ; \psi), \psi\right)=0$, or $\operatorname{Re}\left[\|\psi\|^{2}+\mathrm{i} \lambda k\|\nabla \psi\|^{2}-\mathrm{i} \lambda k\left(f\left(|\varphi|^{2}\right),|\psi|^{2}\right)\right]=\|\psi\|^{2}=0$, which yields $\psi=0$. Hence, we conclude that $T_{h}(\lambda, \varphi ; \cdot)$ is one-to-one. Since $T_{h}(\lambda, \varphi ; \cdot)$ is linear and the space $S_{h}$ has finite dimension, the fact that it is one-to-one yields its invertibility.

Remark 1.3. Taking the $L^{2}$ inner product of (1.8a) with $W_{h}^{1 / 2}+W_{h}^{0}$, of (1.8b) with $W_{h}^{1}+W_{h}^{0}$ and of (1.8c) with $W_{h}^{n}+W_{h}^{n-2}$, and then real parts, we conclude that $\left\|W_{h}^{1 / 2}\right\|=\left\|W_{h}^{0}\right\|$ and $\left\|W_{h}^{\ell}\right\|=\left\|W_{h}^{0}\right\|$ for $\quad \ell=0, \ldots, N$. Thus the method (1.8) is $L^{2}$ conservative.

\subsection{Main results and relations to previous work}

The time discretization in (1.8c) in conjuction with a finite difference method for the space discretization is proposed in [9] for the numerical approximation of a nonlinear Schrödinger equation in one space dimension and with periodic boundary conditions. An optimal order error bound of $\mathcal{O}\left(k^{2}+h^{2}\right)$ in a discrete $L^{2}$ norm is, also, given, only in the case of a cubic Schrödinger equation, where $f(x)=\lambda x$ and $\lambda \in \mathbb{R}$. This convergence result is based on the fact that the method conserves a discrete Hamiltonian which for $d=1$ yields boundedness of the numerical approximations in the discrete $L^{\infty}$ norm by a constant which is independent of the partition of the time and space intervals ( $c f$. (5) in [9] and Rem. 2.22 in Sect. 2.5). In the case of a general nonlinearity this conservation property fails and thus a different technique is needed to prove convergence.

The paper at hand is devoted to the convergence analysis of the method (1.8). We prove an optimal order error bound of $\mathcal{O}\left(k^{2}+h^{r}\right)$ in the $L^{2}$ norm and of $\mathcal{O}\left(k^{2}+h^{r-1}\right)$ in the $H^{1}$ norm, whithout conditions when $d=1$, and under the following mild mesh conditions

$$
\sqrt{|\ln (\underline{h})|} h^{r-1} \leq \mathrm{C}_{2, a} \text { and } \sqrt{|\ln (\underline{h})|}\left(k^{\frac{3}{2}}+h^{r}\right) \leq \mathrm{C}_{2, b} \quad \text { when } d=2,
$$

or

$$
h^{r-1} \leq \mathrm{C}_{3, a} \sqrt{\underline{h}} \text { and } k^{\frac{3}{2}}+h^{r} \leq \mathrm{C}_{3, b} \sqrt{\underline{h}} \quad \text { when } d=3 ;
$$

here $\mathrm{C}_{2, a}, \mathrm{C}_{2, b}, \mathrm{C}_{3, a}$ and $\mathrm{C}_{3, b}$ are constants which depend only on the solution and the data, and $\underline{h}$ is the minimum of the diameter of the elements of the partition of $\Omega$ over which the finite element space is constructed (see Th. 2.14, Th. 2.15 and Rem. 2.16). Also, if $d=2$ and $f$ satisfies $(\mathcal{D})$, then we prove an optimal order error bound $\mathcal{O}\left(k^{2}+h^{r}\right)$ in the $L^{2}$ norm and a suboptimal one of $\mathcal{O}\left(k^{\frac{3}{2}}+h^{r-1}+k^{-\frac{1}{2}} h^{r}\right)$ in the $H^{1}$ norm, provided that

$$
k^{2}+h^{r} \leq \mathrm{C} \sqrt{k}
$$


where $\mathrm{C}$ is a constant which depends only on the solution and the data (see Th. 2.21).

Usually, the analysis of numerical methods for the nonlinear Schrödinger equation is based on inverse inequalities between norms on the finite element spaces for quasiuniform or local quasiuniform partitions of $\Omega$ (see, e.g., $[2,3,11-13,18,20,24])$. Here, when $d=1$, or $d=2$ and $f$ has a polynomial growth satisfying $(\mathcal{D})$, we obtain, for the method (1.8), optimal order of convergence in the $L^{2}$ norm, avoiding inverse inequalities or assumptions on the finite element spaces (as e.g. the $H^{1}$-boundedness assumption of the $L^{2}$-projection $P_{h}$ used in [12]) besides those of Section 1.2. However, for general $f$ and $d=2$ or 3 , we need an inverse inequality between the $L^{\infty}$ and the $H^{1}$ norm, and therefore $\underline{h}$ appears in (1.9) and (1.10).

To arrive at the mesh conditions (1.9), (1.10) or (1.11), we prove convergence estimates in the $L^{2}$ and $H^{1}$ norm, for the approximations generated by a modified scheme which is a nonlinear perturbation of (1.8) at the linearized term (see $(\Lambda)$ and $(\Upsilon)$ in Sect. 2.3). Then the mesh conditions, exhibited above, are introduced to ensure that the modified approximations are bounded in the $L^{\infty}$ or in the $H^{1}$ norm, by a constant independent of the discretization parameters. Having this boundness property the modified scheme coincides with (1.8) and hence the convergence estimates for it hold also for (1.8). The analysis here has been inspired from the works [12] and [24], but there the methods under consideration, the techniques used and the results obtained are different.

The analysis and the results of the paper extend, easily, to the method obtained substituting (1.8c) by

$$
\frac{W_{h}^{n}-W_{h}^{n-2}}{2 k}=\mathrm{i} \Delta_{h}\left(\frac{W_{h}^{n}+W_{h}^{n-2}}{2}\right)+\mathrm{i} P_{h}\left[f\left(\left|W_{h}^{n-1}\right|^{2}\right) W_{h}^{n-1}\right]
$$

which is a nonconservative implicit-explicit method and, as (1.8), yields only one linear system of algebraic equations at every time level, but the matrix remains unchanged.

An overview of the paper is as follows. Section 2 is divided in five parts. In Section 2.1, we prove some function inequalities often used in the convergence analysis, and in Section 2.2 present a consistency result for the time discretization. Section 2.3 contains the definition of the modified schemes and Section 2.4 the convergence theorems for a general function $f$. Finally, in Section 2.5 we investigate the special case where $d=2$ and $f$ satisfies the property $(\mathcal{D})$.

\section{Convergence Analysis}

\subsection{Function inequalities}

We present here some function inequalities that we will often use later.

Lemma 2.1. For $u_{1}, u_{2} \in C(\bar{\Omega})$ and $g \in C^{1}([0,+\infty) ; \mathbb{R})$, we have

$$
\left\|g\left(\left|u_{1}\right|^{2}\right)-g\left(\left|u_{2}\right|^{2}\right)\right\| \leq \sup _{x \in I\left(u_{1}, u_{2}\right)}\left|g^{\prime}(x)\right|\left(\left|u_{1}\right|_{\infty}+\left|u_{2}\right|_{\infty}\right)\left\|u_{1}-u_{2}\right\|
$$

with $I\left(u_{1}, u_{2}\right):=\left[0, \max \left\{\left|u_{1}\right|_{\infty}^{2},\left|u_{2}\right|_{\infty}^{2}\right\}\right]$

Lemma 2.2. For $u_{1}, u_{2}, w_{1}, w_{2} \in C(\bar{\Omega})$ and $g \in C^{2}(\mathbb{R} ; \mathbb{R})$, we have

$$
\begin{aligned}
\left\|g\left(u_{1}\right)-g\left(u_{2}\right)-g\left(w_{1}\right)+g\left(w_{2}\right)\right\| \leq & \sup _{|x| \in I_{1}\left(u_{1}, u_{2}, w_{1}, w_{2}\right)}\left|g^{\prime \prime}(x)\right|\left|w_{1}-w_{2}\right|_{\infty}\left(\left\|u_{1}-w_{1}\right\|+\left\|u_{2}-w_{2}\right\|\right) \\
& +\sup _{|x| \in I_{2}\left(u_{1}, u_{2}\right)}\left|g^{\prime}(x)\right|\left\|\left(u_{1}-u_{2}\right)-\left(w_{1}-w_{2}\right)\right\|,
\end{aligned}
$$

with $I_{1}\left(u_{1}, u_{2}, w_{1}, w_{2}\right):=\left[0, \max \left\{\left|u_{1}\right|_{\infty}+\left|u_{2}\right|_{\infty},\left|w_{1}\right|_{\infty}+\left|w_{2}\right|_{\infty}\right\}\right]$ and $I_{2}\left(u_{1}, u_{2}\right):=\left[0,\left|u_{1}\right|_{\infty}+\left|u_{2}\right|_{\infty}\right]$. 
Proof. (2.2) follows directly from the following expansion

$$
\begin{aligned}
g\left(u_{1}\right)-g\left(u_{2}\right)-g\left(w_{1}\right)+g\left(w_{2}\right)= & \left(w_{1}-w_{2}\right) \int_{0}^{1}\left[g^{\prime}\left((1-\tau) u_{2}+\tau u_{1}\right)-g^{\prime}\left((1-\tau) w_{2}+\tau w_{1}\right)\right] \mathrm{d} \tau \\
& +\left[\left(u_{1}-u_{2}\right)-\left(w_{1}-w_{2}\right)\right] \int_{0}^{1} g^{\prime}\left((1-\tau) u_{2}+\tau u_{1}\right) \mathrm{d} \tau
\end{aligned}
$$

Lemma 2.3. For $u_{1}, u_{2}, w_{1}, w_{2} \in C(\bar{\Omega})$, we have

$$
\begin{aligned}
\left\|\left|u_{1}\right|^{2}-\left|u_{2}\right|^{2}-\left|w_{1}\right|^{2}+\left|w_{2}\right|^{2}\right\| \leq & 2\left|w_{1}-w_{2}\right|_{\infty}\left\|u_{2}-w_{2}\right\| \\
& +B\left(u_{1}, u_{2}, w_{1}, w_{2}\right)\left\|\left(u_{1}-w_{1}\right)-\left(u_{2}-w_{2}\right)\right\|,
\end{aligned}
$$

with $B\left(u_{1}, u_{2}, w_{1}, w_{2}\right):=\max \left\{\left|u_{1}\right|_{\infty}+\left|w_{1}\right|_{\infty},\left|u_{2}\right|_{\infty}+\left|w_{2}\right|_{\infty}\right\}$.

Proof. We obtain (2.3) observing that

$$
\begin{aligned}
\left(\left|u_{1}\right|^{2}-\left|w_{1}\right|^{2}\right)-\left(\left|u_{2}\right|^{2}-\left|w_{2}\right|^{2}\right)= & \operatorname{Re}\left\{\left[\left(u_{1}-w_{1}\right)-\left(u_{2}-w_{2}\right)\right]\left(\overline{u_{1}+w_{1}}\right)\right. \\
& +\left(u_{2}-w_{2}\right)\left[\left(\overline{u_{1}-w_{1}}\right)-\left(\overline{u_{2}-w_{2}}\right)\right] \\
& \left.+2\left(u_{2}-w_{2}\right)\left(\overline{w_{1}-w_{2}}\right)\right\} .
\end{aligned}
$$

Lemma 2.4. Let $F: \mathbb{C}^{2} \longrightarrow \mathbb{C}$ be defined by $F(z, \omega):=\widetilde{f}(|z|) \omega$ where $\widetilde{f}(x):=f\left(x^{2}\right)$ for $x \in \mathbb{R}$. If $d=2$ and $f$ satisfies (1.7), then, for $u_{1}, u_{2}, w_{1}, w_{2} \in C(\bar{\Omega})$ with $u_{1}-w_{1}, u_{2}-w_{2} \in H_{0}^{1}(\Omega)$, we have

$$
\left\|F\left(u_{1}, u_{2}\right)-F\left(w_{1}, w_{2}\right)\right\| \leq C\left(\left\|u_{1}-w_{1}\right\|+\left\|u_{2}-w_{2}\right\|\right)\left[1+\sum_{j=1}^{2}\left(\left|w_{j}\right|_{\infty}^{\varrho}+\left\|\nabla\left(u_{j}-w_{j}\right)\right\|^{\varrho}\right)\right] .
$$

Proof. Let $u_{1}, u_{2}, w_{1}, w_{2} \in C(\bar{\Omega})$ with $u_{1}-w_{1}, u_{2}-w_{2} \in H_{0}^{1}(\Omega)$. Observing that

$$
\left|F\left(z_{1}, z_{2}\right)-F\left(\omega_{1}, \omega_{2}\right)\right| \leq C\left(\left|z_{1}-\omega_{1}\right|+\left|z_{2}-\omega_{2}\right|\right)\left[1+\left(\left|\omega_{1}\right|+\left|\omega_{2}\right|+\left|z_{1}-\omega_{1}\right|+\left|z_{2}-\omega_{2}\right|\right)^{\varrho}\right]
$$

for $z_{1}, z_{2}, \omega_{1}, \omega_{2} \in \mathbb{C}$, and using (1.4), we obtain

$$
\begin{aligned}
\left\|F\left(u_{1}, u_{2}\right)-F\left(w_{1}, w_{2}\right)\right\| \leq C\left[\int_{\Omega}\left[1+\left(\left|w_{1}\right|+\left|w_{2}\right|\right)^{2 \varrho}\right]\left(\left|u_{1}-w_{1}\right|+\left|u_{2}-w_{2}\right|\right)^{2} \mathrm{~d} x\right. & \\
& \left.+\int_{\Omega}\left(\left|u_{1}-w_{1}\right|+\left|u_{2}-w_{2}\right|\right)^{2 \varrho+2} \mathrm{~d} x\right]^{1 / 2} \\
\leq & C\left[\left(1+\left|w_{1}\right|_{\infty}^{2 \varrho}+\left|w_{2}\right|_{\infty}^{2 \varrho}\right)\left(\left\|u_{1}-w_{1}\right\|^{2}+\left\|u_{2}-w_{2}\right\|^{2}\right)\right. \\
& \left.+\left\|u_{1}-w_{1}\right\|^{2}\left\|\nabla\left(u_{1}-w_{1}\right)\right\|^{2 \varrho}+\left\|u_{2}-w_{2}\right\|^{2}\left\|\nabla\left(u_{2}-w_{2}\right)\right\|^{2 \varrho}\right]^{1 / 2}
\end{aligned}
$$

which yields (2.4). 


\subsection{Consistency}

We continue by presenting a consistency result concerning the time discretization.

For $n=0, \ldots, N$, we define $\sigma^{n} \in L^{2}(\Omega)$ by

$$
\frac{w^{\ell_{n}^{*}}-w^{j_{n}^{*}}}{\lambda_{n} k}=\frac{\mathrm{i}}{2} \Delta\left(w^{\ell_{n}^{*}}+w^{j_{n}^{*}}\right)+\frac{\mathrm{i}}{2} f\left(\left|w^{i_{n}^{*}}\right|^{2}\right)\left(w^{\ell_{n}^{*}}+w^{j_{n}^{*}}\right)+\sigma^{n}
$$

where $w^{1 / 2}:=w\left(\cdot, t^{1 / 2}\right), w^{m}:=w\left(\cdot, t^{m}\right)$ for $m=0, \ldots, N, \lambda_{0}:=1 / 2, \lambda_{1}:=1, \lambda_{m}:=2$ for $m=2, \ldots, N$, $\ell_{0}^{*}:=1 / 2, \ell_{m}^{*}:=m$ for $m=1, \ldots, N, i_{0}^{*}:=0, i_{1}^{*}:=1 / 2, i_{m}^{*}:=m-1$ for $m=2, \ldots, N$, and $j_{0}^{*}:=0, j_{1}^{*}:=0$, $j_{m}^{*}:=m-2$ for $m=2, \ldots, N$. Then, using Taylor expansions, we arrive at

$$
\max _{1 \leq n \leq N}\left\|\sigma^{n}\right\|+k\left\|\sigma^{0}\right\| \leq C k^{2} \text { and } \max _{4 \leq n \leq N}\left\|\sigma^{n}-\sigma^{n-2}\right\| \leq C k^{3} .
$$

\subsection{Modified Schemes}

Modifying properly the linearized term in the numerical method (1.8), we construct two modified schemes, $(\Lambda)$ and $(\Upsilon)$, which we will use later in the convergence analysis. A modified scheme is connected to a real parameter $\delta>0$ and a given norm of $\mathcal{H}$, and it is not a numerical method. When the approximations that the scheme furnishes are bounded in that norm by $\delta$, then they coincide with those that (1.8) produces provided, of course, that the initial approximation is the same. Even that the original method (1.8) is linear, the modified scheme will be nonlinear. Hence, we cannot ensure the existence of the modified approximations following the argument of Remark 1.2. For this reason, we shall employ the following Brouwer-type fixed-point lemma, for a proof of which we refer to [3].

Lemma 2.5. Let $\left(\mathcal{X},(\cdot, \cdot)_{\mathcal{X}}\right)$ be a finite-dimensional inner product space and $\|\cdot\|_{\mathcal{X}}$ the associated norm. Let $\mu: \mathcal{X} \longrightarrow \mathcal{X}$ be continuous and assume that there exists $\alpha>0$ such that for every $z \in \mathcal{X}$ with $\|z\|_{\mathcal{X}}=\alpha$ there holds $\operatorname{Re}(\mu(z), z)_{\mathcal{X}} \geq 0$. Then, there exists a $z^{*} \in \mathcal{X}$ such that $\mu\left(z^{*}\right)=0$ and $\left\|z^{*}\right\|_{\mathcal{X}} \leq \alpha$.

- Modified Scheme $(\Lambda)$ : Let $\delta>\sup _{t \in I_{*}}|w(\cdot, t)|_{\infty}$ and $g_{\delta}$ be an increasing $C^{2}(\mathbb{R} ; \mathbb{R})$ function, with bounded derivatives up to second order, satisfying

$$
g_{\delta}(x):=\left\{\begin{array} { l l } 
{ x , } & { \text { if } \quad | x | \leq \delta } \\
{ 2 \frac { x } { | x | } \delta , } & { \text { if } \quad | x | > 2 \delta }
\end{array} \quad \text { and } g _ { \delta } ( x ) \in \left\{\begin{array}{ll}
{[\delta, 2 \delta]} & \text { if } \quad x \in[\delta, 2 \delta] \\
{[-2 \delta,-\delta]} & \text { if } \quad x \in[-2 \delta,-\delta]
\end{array} \quad \text { for } \quad x \in \mathbb{R} .\right.\right.
$$

Then, we define a function $\gamma_{\delta}: \mathbb{C} \longrightarrow \mathbb{C}$ by $\gamma_{\delta}(z)=g_{\delta}(\operatorname{Re} z)+\mathrm{i} g_{\delta}(\operatorname{Im} z)$.

For $h \in(0,1)$ and $m=0,1 / 2,1, \ldots, N$, let $\Lambda_{\delta, h}^{m} \in S_{h}$ be specified inductively by

$$
\Lambda_{\delta, h}^{0}=R_{h} w^{0}
$$

and

$$
\frac{\Lambda_{\delta, h}^{\ell_{m}^{*}}-\Lambda_{\delta, h}^{j_{m}^{*}}}{\lambda_{m} k}=\mathrm{i} \Delta_{h}\left(\frac{\Lambda_{\delta, h}^{\ell_{m}^{*}}+\Lambda_{\delta, h}^{j_{m}^{*}}}{2}\right)+\mathrm{i} P_{h}\left[f\left(\left|\gamma_{\delta}\left(\Lambda_{\delta, h}^{i_{m}^{*}}\right)\right|^{2}\right) \gamma_{\delta}\left(\frac{\Lambda_{\delta, h}^{\ell_{m}^{*}}+\Lambda_{\delta, h}^{j_{m}^{*}}}{2}\right)\right]
$$

for $m=0, \ldots, N$.

Remark 2.6. It is easily seen that $\gamma_{\delta}(w(\cdot, \tau))=w(\cdot, \tau)$ and $\gamma_{\delta}\left(\frac{w\left(\cdot, \tau_{1}\right)+w\left(\cdot, \tau_{2}\right)}{2}\right)=\frac{w\left(\cdot, \tau_{1}\right)+w\left(\cdot, \tau_{2}\right)}{2}$ for $\tau, \tau_{1}, \tau_{2} \in I_{*}$, where $w$ is the solution of (1.1). Thus, the consistency argument for the method (1.8) (cf. Sect. 2.2) holds also for $(2.7)$. 
Remark 2.7. Assuming that $\left|\Lambda_{\delta, h}^{0}\right|_{\infty} \leq \delta$ and $\left|\Lambda_{\delta, h}^{\ell_{m}^{*}}\right|_{\infty} \leq \delta$ for $m=0, \ldots, N$, we obtain $f\left(\left|\gamma_{\delta}\left(\Lambda_{\delta, h}^{i_{m}^{*}}\right)\right|^{2}\right)=$ $f\left(\left|\Lambda_{\delta, h}^{i_{m}^{*}}\right|^{2}\right)$ and $\gamma_{\delta}\left(\frac{\Lambda_{\delta, h}^{\ell_{*}^{*}}+\Lambda_{\delta, h}^{j_{*}^{*}}}{2}\right)=\frac{\Lambda_{\delta, h}^{\ell^{*}}+\Lambda_{\delta, h}^{j_{*}^{*}}}{2}$ for $m=0, \ldots, N$. Hence, if $W_{h}^{0}=R_{h} w^{0}$, then $\Lambda_{\delta, h}^{\ell_{m}^{*}}=W_{h}^{\ell_{m}^{*}}$ for $m=0, \ldots, N$.

We ensure the existence of a $\Lambda_{\delta, h}^{\ell_{m}^{*}} \in S_{h}$ which solves the nonlinear system in (2.7b), by an argument based on Lemma 2.5. In particular, let $m \in\{0, \ldots, N\}, h \in(0,1),\left(\mathcal{X},(\cdot, \cdot)_{\mathcal{X}}\right)=\left(S_{h},(\cdot, \cdot)\right)$ and $\Pi_{\delta, h}^{m}: \mathcal{X} \longrightarrow \mathcal{X}$ be an operator given by

$$
\Pi_{\delta, h}^{m}(\chi):=\chi-\Lambda_{\delta, h}^{j_{m}^{*}}-\frac{\mathrm{i}}{2} \lambda_{m} k \Delta_{h} \chi-\frac{\mathrm{i}}{2} \lambda_{m} k P_{h}\left[f\left(\left|\gamma_{\delta}\left(\Lambda_{\delta, h}^{i_{m}^{*}}\right)\right|^{2}\right) \gamma_{\delta}(\chi)\right]
$$

$\Pi_{\delta, h}^{m}$ is continuous, since $\gamma_{\delta}$ is continuous and $S_{h}$ has finite dimension. Then, we obtain

$$
\operatorname{Re}\left(\Pi_{\delta, h}^{m}(\chi), \chi\right) \geq\|\chi\|\left(\|\chi\|-\left\|\Lambda_{\delta, h}^{j_{m}^{*}}\right\|-\lambda_{m} k B_{\delta}\right), \quad \forall \chi \in S_{h},
$$

where $B_{\delta}:=\sqrt{2} \delta|\Omega|^{1 / 2} \sup _{x \in\left[0,8 \delta^{2}\right]}|f(x)|$ and $|\Omega|$ is the area of $\Omega$. Therefore, we have $\operatorname{Re}\left(\Pi_{\delta, h}^{m}(\chi), \chi\right)>0$ for every $\chi \in S_{h}$ with $\|\chi\|=1+\left\|\Lambda_{\delta, h}^{j_{m}^{*}}\right\|+\lambda_{m} k B_{\delta}$. Applying Lemma 2.5 with $\mu=\Pi_{\delta, h}^{m}$, we conclude that there exists a $\chi_{\delta, h}^{m} \in S_{h}$ such that $\Pi_{\delta, h}^{m}\left(\chi_{\delta, h}^{m}\right)=0$. Thus $\Lambda_{\delta, h}^{\ell_{m}^{*}}=2 \chi_{\delta, h}^{m}-\Lambda_{\delta, h}^{j_{m}^{*}}$ is a solution of $(2.7 \mathrm{~b})$.

In Remark 2.7, we explained that when the approximations produced by $(\Lambda)$ are bounded in the $L^{\infty}$ norm by $\delta$ and $W_{h}^{0}=R_{h} w^{0}$, then they are the numerical approximations of the method (1.8). Next, we present another modified scheme that has this property for any norm $\nu$ on $\mathcal{H}$, instead of the $L^{\infty}$ one.

- Modified Scheme $(\Upsilon)$ : Let $\nu$ be a norm on $\mathcal{H}, \delta>\sup _{t \in I_{*}} \nu(w(\cdot, t))$ be a given constant, and $\xi_{\delta}: \mathbb{R} \longrightarrow \mathbb{R}$ be a continuous function defined by

$$
\xi_{\delta}(x):=\left\{\begin{array}{lll}
1, & \text { if } & x \leq 2 \delta \\
-\frac{x}{\delta}+3, & \text { if } & x \in[2 \delta, 3 \delta] \quad \text { for } x \in \mathbb{R} . \\
0, & \text { if } & x>3 \delta
\end{array}\right.
$$

Then, for $t \in I_{*}$, we define a map $g_{\nu, \delta}(t ; \cdot): \mathcal{H} \longrightarrow \mathcal{H}$ by

$$
g_{\nu, \delta}(t ; \omega):=\omega \xi_{\delta}(\nu(\omega-w(\cdot, t)))+w(\cdot, t)\left(1-\xi_{\delta}(\nu(\omega-w(\cdot, t)))\right) \text { for } \omega \in \mathcal{H},
$$

where $w$ is, always, the solution of problem (1.1).

For $h \in(0,1)$ and $m=0,1 / 2,1, \ldots, N$, we specify functions $\Upsilon_{\delta, h}^{m} \in S_{h}$, inductively by

$$
\Upsilon_{\delta, h}^{0}=R_{h} w^{0}
$$

and

$$
\frac{\Upsilon_{\delta, h}^{\ell_{m}^{*}}-\Upsilon_{\delta, h}^{j_{m}^{*}}}{\lambda_{m} k}=\mathrm{i} \Delta_{h}\left(\frac{\Upsilon_{\delta, h}^{\ell_{m}^{*}}+\Upsilon_{\delta, h}^{j_{m}^{*}}}{2}\right)+\mathrm{i} P_{h}\left[f\left(\left|g_{\nu, \delta}\left(t^{i_{m}^{*}} ; \Upsilon_{\delta, h}^{i_{m}^{*}}\right)\right|^{2}\right) \frac{g_{\nu, \delta}\left(t_{m}^{\ell_{m}^{*}} ; \Upsilon_{\delta, h}^{\ell_{m}^{*}}\right)+g_{\nu, \delta}\left(t_{m}^{j_{m}^{*}} ; \Upsilon_{\delta, h}^{j_{m}^{*}}\right)}{2}\right]
$$

for $m=0, \ldots, N$.

Remark 2.8. Since $g_{\nu, \delta}(t ; w(\cdot, t))=w(\cdot, t)$ for $t \in I_{*}$, the consistency argument for $(2.8)$ is the same with that for (1.8) (cf. Sect. 2.2).

Remark 2.9. Assuming that $\nu\left(\Upsilon_{\delta, h}^{0}\right) \leq \delta$ and $\nu\left(\Upsilon_{\delta, h}^{\ell_{m}^{*}}\right) \leq \delta$ for $m=0, \ldots, N$, we have $\nu\left(\Upsilon_{\delta, h}^{0}-w(\cdot, t)\right)<2 \delta$ and $\nu\left(\Upsilon_{\delta, h}^{\ell_{m}^{*}}-w(\cdot, t)\right) \leq 2 \delta$ for $m=0, \ldots, N$ and $t \in I_{*}$. Thus, we obtain $g_{\nu, \delta}\left(t ; \Upsilon_{\delta, h}^{\ell_{m}^{*}}\right)=\Upsilon_{\delta, h}^{\ell_{m}^{*}}$ for $m=0, \ldots, N$ and $t \in I_{*}$. In that case, if $W_{h}^{0}=R_{h} w^{0}$, then a simple induction argument yields $\Upsilon_{\delta, h}^{\ell_{m}^{*}}=W_{h}^{\ell_{m}^{*}}$ for $m=0, \ldots, N$. 
Lemma 2.10. Let $\nu$ be a norm on $\mathcal{H}$ and $\delta>\sup _{t \in I_{*}} \nu(w(\cdot, t))$. Then, for $t \in I_{*}$ and $\omega \in \mathcal{H}$, we have

$$
\nu\left(g_{\nu, \delta}(t ; \omega)\right) \leq 4 \delta
$$

Proof. Let $t \in I_{*}$ and $\omega \in \mathcal{H}$. If $\nu(\omega-w(\cdot, t)) \geq 3 \delta$ then $\nu\left(g_{\nu, \delta}(t ; \omega)\right)=\nu(w(\cdot, t)) \leq \delta$. We assume, now, that $\nu(\omega-w(\cdot, t))<3 \delta$. Then we have $\nu(\omega) \leq 4 \delta$, and

$$
\begin{aligned}
\nu\left(g_{\nu, \delta}(t ; \omega)\right) & \leq \nu(\omega) \xi_{\delta}(\nu(\omega-w(\cdot, t)))+\nu(w(\cdot, t))\left(1-\xi_{\delta}(\nu(\omega-w(\cdot, t)))\right) \\
& \leq 3 \delta \xi_{\delta}(\nu(\omega-w(\cdot, t)))+\delta \\
& \leq 4 \delta
\end{aligned}
$$

Lemma 2.11. Let $\nu$ be a norm on $\mathcal{H}$ and $\delta>\sup _{t \in I_{*}} \nu(w(\cdot, t))$. For $t \in I_{*}$, the operator $g_{\nu, \delta}(t ; \cdot): \mathcal{H} \longrightarrow \mathcal{H}$ is continuous on $(\mathcal{H}, \nu)$.

Proof. For $t \in I_{*}$ and $\omega_{1}, \omega_{2} \in \mathcal{H}$, we have

$$
\nu\left(g_{\nu, \delta}\left(t ; \omega_{1}\right)-g_{\nu, \delta}\left(t ; \omega_{2}\right)\right) \leq \nu\left(\omega_{1}-\omega_{2}\right)+\left[\nu(w(\cdot, t))+\nu\left(\omega_{2}\right)\right]\left|\xi_{\delta}\left(\nu\left(\omega_{1}-w(\cdot, t)\right)\right)-\xi_{\delta}\left(\nu\left(\omega_{2}-w(\cdot, t)\right)\right)\right|
$$

which, together with the continuity of $\xi_{\delta}$, yields the continuity of $g_{\nu, \delta}(t ; \cdot)$ on $(\mathcal{H}, \nu)$.

As for $(\Lambda)$, we discuss, now, the existence of a solution for the nonlinear system in (2.8b) following a similar argument. Let $m \in\{0, \ldots, N\}, h \in(0,1),\left(\mathcal{X},(\cdot, \cdot)_{\mathcal{X}}\right)=\left(S_{h},(\cdot, \cdot)\right)$ and $\Phi_{\delta, h}^{m}: \mathcal{X} \longrightarrow \mathcal{X}$ given by

$$
\Phi_{\delta, h}^{m}(\chi):=\chi-\Upsilon_{\delta, h}^{j_{m}^{*}}-\frac{\mathrm{i}}{2} \lambda_{m} k \Delta_{h} \chi-\frac{\mathrm{i}}{4} \lambda_{m} k P_{h}\left[f\left(\left|g_{\nu, \delta}\left(t_{m}^{i_{m}^{*}} ; \Upsilon_{\delta, h}^{i_{m}^{*}}\right)\right|^{2}\right)\left[g_{\nu, \delta}\left(t_{m}^{\ell_{m}^{*}} ; 2 \chi-\Upsilon_{\delta, h}^{j_{m}^{*}}\right)+g_{\nu, \delta}\left(t^{j_{m}^{*}} ; \Upsilon_{\delta, h}^{j_{m}^{*}}\right)\right]\right]
$$

which is continuous, since $g_{\nu, \delta}\left(t_{m}^{\ell_{m}^{*}} ; \cdot\right)$ is continuous ( $f f$. Lem. 2.11) and $S_{h}$ has finite dimension. Also, in $S_{h}$ the norms $\nu$ and $|\cdot|_{\infty}$ are equivalent, and hence there exists an $h$-dependent constant $C_{h, \infty, \nu}$, such that $|\chi|_{\infty} \leq C_{h, \infty, \nu} \nu(\chi)$ for $\chi \in S_{h}$. Using (2.9), we arrive at the following general estimates

$$
\left|g_{\nu, \delta}(\tau ; \omega)\right|_{\infty} \leq \widetilde{B}_{1, h, \delta}:=4 \delta C_{h, \infty, \nu}, \quad \forall \tau \in I_{*}, \quad \forall \omega \in \mathcal{H},
$$

and

$$
\left\|f\left(\left|g_{\nu, \delta}\left(\tau_{1} ; \omega_{1}\right)\right|^{2}\right)\left[g_{\nu, \delta}\left(\tau_{2} ; \omega_{2}\right)+g_{\nu, \delta}\left(\tau_{3} ; \omega_{3}\right)\right]\right\| \leq \widetilde{B}_{2, h, \delta}:=2 \widetilde{B}_{1, h, \delta} \sqrt{|\Omega|} \sup _{\left[0,\left(\widetilde{B}_{1, h, \delta}\right)^{2}\right]}|f|,
$$

for $\tau_{1}, \tau_{2}, \tau_{3} \in I_{*}$ and $\omega_{1}, \omega_{2}, \omega_{3} \in \mathcal{H}$. Thus, we obtain

$$
\operatorname{Re}\left(\Phi_{\delta, h}^{m}(\chi), \chi\right) \geq\|\chi\|\left(\|\chi\|-\left\|\Upsilon_{\delta, h}^{j_{m}^{*}}\right\|-\frac{\lambda_{m} k}{4} \widetilde{B}_{2, h, \delta}\right), \quad \forall \chi \in S_{h}
$$

Therefore, we have $\operatorname{Re}\left(\Phi_{\delta, h}^{m}(\chi), \chi\right)>0$ for every $\chi \in S_{h}$ with $\|\chi\|=1+\left\|\Upsilon_{\delta, h}^{j_{m}^{*}}\right\|+\frac{\lambda_{m} k}{4} \widetilde{B}_{2, h, \delta}$, and by Lemma 2.5, with $\mu=\Phi_{\delta, h}^{m}$, we conclude the existence of a $\chi_{\delta, h}^{m} \in S_{h}$ such that $\Phi_{\delta, h}^{m}\left(\chi_{\delta, h}^{m}\right)=0$. Finally, $\Upsilon_{\delta, h}^{\ell_{m}^{*}}=2 \chi_{\delta, h}^{m}-\Upsilon_{\delta, h}^{j_{m}^{*}}$ is a solution of $(2.8 \mathrm{~b})$. 


\section{4. $L^{2}$ - and $H^{1}$-convergence}

Next we will prove the following optimal order error estimates for the method (1.8):

$$
\max _{0 \leq m \leq N}\left\|W_{h}^{m}-w^{m}\right\| \leq \mathcal{C}_{A}\left(k^{2}+h^{r}\right) \text { and } \max _{0 \leq m \leq N}\left\|\nabla\left(W_{h}^{m}-w^{m}\right)\right\| \leq \mathcal{C}_{A}\left(k^{2}+h^{r-1}\right)
$$

for $h \in\left(0, h_{0}\right)$ and $N \geq N_{0}$. Here $h_{0} \in(0,1), N_{0} \in \mathbb{N}$ and $\mathcal{C}_{A}$ is a constant independent of $h$ and $N$.

The basic step in the convergence analysis is the estimation of the differences $\vartheta_{\Lambda, \delta, h}^{1 / 2}:=\Lambda_{\delta, h}^{1 / 2}-R_{h} w^{1 / 2}$ and $\vartheta_{\Lambda, \delta, h}^{n}:=\Lambda_{\delta, h}^{n}-R_{h} w^{n}, n=0, \ldots, N$, where due to $(2.7 \mathrm{a})$, we have $\vartheta_{\Lambda, \delta, h}^{0}=0$.

Proposition 2.12. Let $d=1,2$ or 3 , and $\delta>\sup _{t \in I_{*}}|w(\cdot, t)|_{\infty}$. Then, there exists $N_{\delta} \in \mathbb{N}$ such that

$$
\left\|\vartheta_{\Lambda, \delta, h}^{1 / 2}\right\| \leq C_{A, \delta}\left(k^{2}+k h^{r}\right) \quad \text { and } \max _{1 \leq m \leq N}\left\|\vartheta_{\Lambda, \delta, h}^{m}\right\| \leq C_{A, \delta}\left(k^{2}+h^{r}\right), \quad \forall h \in(0,1), \quad \forall N \geq N_{\delta}
$$

The constant $C_{A, \delta}$ is independent of $h$ and $N$, but depends on $\delta$, the solution or its derivatives, and the data.

Proof. Let $h \in(0,1)$. For $m=0, \ldots, N$, we define $\sigma_{\Lambda, \delta, h}^{m} \in S_{h}$ by

$$
\frac{\vartheta_{\Lambda, \delta, h}^{\ell_{m}^{*}}-\vartheta_{\Lambda, \delta, h}^{j_{m}^{*}}}{\lambda_{m} k}=\mathrm{i} \Delta_{h}\left(\frac{\vartheta_{\Lambda, \delta, h}^{\ell_{m}^{*}}+\vartheta_{\Lambda, \delta, h}^{j_{m}^{*}}}{2}\right)+\sigma_{\Lambda, \delta, h}^{m} .
$$

Combining (2.12), (2.5), Remark 2.6, and (1.8), we obtain

$$
\sigma_{\Lambda, \delta, h}^{m}=-P_{h}\left(\sigma^{m}+\omega_{w}^{m}\right)+\mathrm{i} P_{h} \omega_{\Lambda, \delta}^{m}, \quad m=0, \ldots, N
$$

with

$$
\omega_{w}^{m}:=R_{h}\left(\frac{w^{\ell_{m}^{*}}-w^{j_{m}^{*}}}{\lambda_{m} k}\right)-\left(\frac{w^{\ell_{m}^{*}}-w^{j_{m}^{*}}}{\lambda_{m} k}\right)
$$

and

$$
\omega_{\Lambda, \delta}^{m}:=f\left(\left|\gamma_{\delta}\left(\Lambda_{\delta, h}^{i_{m}^{*}}\right)\right|^{2}\right) \gamma_{\delta}\left(\frac{\Lambda_{\delta, h}^{\ell_{m}^{*}}+\Lambda_{\delta, h}^{j_{m}^{*}}}{2}\right)-f\left(\left|w_{m}^{i_{m}^{*}}\right|^{2}\right) \gamma_{\delta}\left(\frac{w^{\ell_{m}^{*}}+w^{j_{m}^{*}}}{2}\right)
$$

Taking real parts of the $L^{2}$ inner product of (2.12) with $\vartheta_{h}^{\ell_{m}^{*}}+\vartheta_{h}^{j_{m}^{*}}$ and using $(2.13)$, for $m=0, \ldots, N$, we have

$$
\left\|\vartheta_{\Lambda, \delta, h}^{\ell_{m}^{*}}\right\|^{2}-\left\|\vartheta_{\Lambda, \delta, h}^{j_{m}^{*}}\right\|^{2}=-\lambda_{m} k\left[\operatorname{Re}\left(\sigma^{m}+\omega_{w}^{m}, \vartheta_{\Lambda, \delta, h}^{\ell_{m}^{*}}+\vartheta_{\Lambda, \delta, h}^{j_{m}^{*}}\right)+\operatorname{Im}\left(\omega_{\Lambda, \delta}^{m}, \vartheta_{\Lambda, \delta, h}^{\ell_{m}^{*}}+\vartheta_{\Lambda, \delta, h}^{j_{m}^{*}}\right)\right]
$$

By (1.5) and the Taylor formula, we conclude that

$$
\max _{0 \leq m \leq N}\left\|\omega_{w}^{m}\right\| \leq C h^{r}
$$

Let $m \in\{0, \ldots, N\}$. To estimate $\omega_{\Lambda, \delta}^{m}$ in the $L^{2}$ norm, we split it as follows:

$$
\omega_{\Lambda, \delta}^{m}=\omega_{\Lambda, \delta, 1}^{m}+\omega_{\Lambda, \delta, 2}^{m}
$$


with

$$
\omega_{\Lambda, \delta, 1}^{m}:=\left[f\left(\left|\gamma_{\delta}\left(\Lambda_{\delta, h}^{i_{m}^{*}}\right)\right|^{2}\right)-f\left(\left|w^{i_{m}^{*}}\right|^{2}\right)\right] \gamma_{\delta}\left(\frac{\Lambda_{\delta, h}^{e_{m}^{*}}+\Lambda_{\delta, h}^{j_{m}^{*}}}{2}\right) \text { and } \omega_{\Lambda, \delta, 2}^{m}:=f\left(\left|w^{i_{m}^{*}}\right|^{2}\right)\left[\gamma_{\delta}\left(\frac{\Lambda_{\delta, h}^{e_{m}^{*}}+\Lambda_{\delta, h}^{j_{m}^{*}}}{2}\right)-\gamma_{\delta}\left(\frac{w_{m}^{e_{m}^{*}}+w^{j_{m}^{*}}}{2}\right)\right] \text {. }
$$

Using (2.1), the mean value theorem and (1.5), we obtain

$$
\begin{aligned}
\left\|\omega_{\Lambda, \delta, 1}^{m}\right\| & \leq C \delta(1+\delta) \sup _{x \in\left[0,8 \delta^{2}\right]}\left|f^{\prime}(x)\right|\left\|\gamma_{\delta}\left(\Lambda_{\delta, h}^{i_{m}^{*}}\right)-\gamma_{\delta}\left(w^{i_{m}^{*}}\right)\right\| \\
& \leq C_{\delta} \sup _{|x| \in[0,2 \delta]}\left|g_{\delta}^{\prime}(x)\right|\left(h^{r}+\left\|\vartheta_{\Lambda, \delta, h}^{i_{m}^{*}}\right\|\right)
\end{aligned}
$$

and

$$
\left\|\omega_{\Lambda, \delta, 2}^{m}\right\| \leq C \sup _{|x| \in[0,2 \delta]}\left|g_{\delta}^{\prime}(x)\right|\left(h^{r}+\left\|\vartheta_{\Lambda, \delta, h}^{\ell_{m}^{*}}\right\|+\left\|\vartheta_{\Lambda, \delta, h}^{j_{m}^{*}}\right\|\right) .
$$

Thus, we get the following estimate

$$
\left\|\omega_{\Lambda, \delta}^{m}\right\| \leq C_{\delta}\left(h^{r}+\left\|\vartheta_{\Lambda, \delta, h}^{\ell_{m}^{*}}\right\|+\left\|\vartheta_{\Lambda, \delta, h}^{j_{m}^{*}}\right\|+\left\|\vartheta_{\Lambda, \delta, h}^{i_{m}^{*}}\right\|\right) .
$$

From (2.16), (2.17), (2.6) and (2.18), it follows that

$$
\left\|\vartheta_{\Lambda, \delta, h}^{1 / 2}\right\|+\left\|\vartheta_{\Lambda, \delta, h}^{1}\right\| \leq \widetilde{C}_{\delta} k\left(\left\|\vartheta_{\Lambda, \delta, h}^{1 / 2}\right\|+\left\|\vartheta_{\Lambda, \delta, h}^{1}\right\|\right)+C_{\delta}\left(k^{2}+k h^{r}\right)
$$

and

$$
\left(1-\widetilde{C}_{\delta} k\right)\left(\left\|\vartheta_{\Lambda, \delta, h}^{m}\right\|+\left\|\vartheta_{\Lambda, \delta, h}^{m-1}\right\|\right) \leq\left(1+\widetilde{C}_{\delta} k\right)\left(\left\|\vartheta_{\Lambda, \delta, h}^{m-1}\right\|+\left\|\vartheta_{\Lambda, \delta, h}^{m-2}\right\|\right)+C_{\delta} k\left(k^{2}+h^{r}\right), \quad m=2, \ldots, N .
$$

Finally, assuming that $\widetilde{C}_{\delta} k \leq \frac{1}{3}$ and applying a discrete Grönwall argument on (2.19a-b), we arrive at (2.11).

Proposition 2.13. Let $d=1,2$ or 3 , and $\delta>\sup _{t \in I_{*}}|w(\cdot, t)|_{\infty}$. Then there exists an integer $\widetilde{N}_{\delta} \geq N_{\delta}$ such that

$$
\left\|\nabla \vartheta_{\Lambda, \delta, h}^{1 / 2}\right\| \leq \mathcal{C}_{A, \delta}\left(k^{\frac{3}{2}}+k^{\frac{1}{2}} h^{r}\right) \quad \text { and } \max _{1 \leq m \leq N}\left\|\nabla \vartheta_{\Lambda, \delta, h}^{m}\right\| \leq \mathcal{C}_{A, \delta}\left(k^{2}+h^{r}\right), \quad \forall h \in(0,1), \quad \forall N \geq \widetilde{N}_{\delta} .
$$

The constant $\mathcal{C}_{A, \delta}$ is independent of $h$ and $N$, but depends on $\delta$, the solution or its derivatives, and the data.

Proof. Let $h \in(0,1)$ and $N \geq N_{\delta}$ (cf. Prop. 2.12). For $m=0, \ldots, N$, we define $\sigma_{\Lambda, \delta, h}^{m}, \omega_{w}^{m}$ and $\omega_{\Lambda, \delta}^{m}$ as in (2.12), (2.14) and (2.15), respectively.

Taking the $L^{2}$-inner product of $(2.12)$ by $\vartheta_{\Lambda, \delta, h}^{\ell_{m}^{*}}-\vartheta_{\Lambda, \delta, h}^{j_{m}^{*}}$ and then imaginary parts, we obtain

$$
\left\|\nabla \vartheta_{\Lambda, \delta, h}^{1 / 2}\right\|^{2}=2 \operatorname{Im}\left(\sigma_{\Lambda, \delta, h}^{0}, \vartheta_{\Lambda, \delta, h}^{1 / 2}\right), \quad\left\|\nabla \vartheta_{\Lambda, \delta, h}^{1}\right\|^{2}=2 \operatorname{Im}\left(\sigma_{\Lambda, \delta, h}^{1}, \vartheta_{\Lambda, \delta, h}^{1}\right)
$$

and

$$
\left\|\nabla \vartheta_{\Lambda, \delta, h}^{m}\right\|^{2}+\left\|\nabla \vartheta_{\Lambda, \delta, h}^{m-1}\right\|^{2}=\left\|\nabla \vartheta_{\Lambda, \delta, h}^{m-1}\right\|^{2}+\left\|\nabla \vartheta_{\Lambda, \delta, h}^{m-2}\right\|^{2}+2 \operatorname{Im}\left(\sigma_{\Lambda, \delta, h}^{m}, \vartheta_{\Lambda, \delta, h}^{m}-\vartheta_{\Lambda, \delta, h}^{m-2}\right), \quad m=2, \ldots, N
$$


Hence, summing with respect to $m$ in $(2.22)$, it follows that

$$
\begin{aligned}
\left\|\nabla \vartheta_{\Lambda, \delta, h}^{n}\right\|^{2}+\left\|\nabla \vartheta_{\Lambda, \delta, h}^{n-1}\right\|^{2} \leq & \left\|\nabla \vartheta_{\Lambda, \delta, h}^{1}\right\|^{2}+\left\|\nabla \vartheta_{\Lambda, \delta, h}^{0}\right\|^{2} \\
& +\max _{2 \leq m \leq N}\left\|\sigma_{\Lambda, \delta, h}^{m}\right\|^{2}+\left(\sum_{m=2}^{n}\left\|\vartheta_{\Lambda, \delta, h}^{m}-\vartheta_{\Lambda, \delta, h}^{m-2}\right\|^{2}, \quad n=2, \ldots, N .\right.
\end{aligned}
$$

Next, we will estimate the quantities at the right-hand side of (2.23).

- Estimation of $\left\|\sigma_{\Lambda, \delta, h}^{m}\right\|$ : From (2.18) and (2.11), we obtain

$$
\max _{0 \leq m \leq N}\left\|\omega_{\Lambda, \delta}^{m}\right\| \leq C_{\delta}\left(k^{2}+h^{r}\right)
$$

which, together with (2.13), (2.6) and (2.17), implies

$$
\left\|\sigma_{\Lambda, \delta, h}^{0}\right\| \leq C_{\delta}\left(k+h^{r}\right) \text { and } \max _{1 \leq m \leq N}\left\|\sigma_{\Lambda, \delta, h}^{m}\right\| \leq C_{\delta}\left(k^{2}+h^{r}\right) .
$$

- Estimation of $\left\|\omega_{w}^{m}-\omega_{w}^{m-2}\right\|$ : Using (1.5), we have

$$
\begin{aligned}
\left\|\omega_{w}^{m}-\omega_{w}^{m-2}\right\| & \leq C \frac{h^{r}}{2 k}\left\|w^{m}-2 w^{m-2}+w^{m-4}\right\|_{r} \\
& \leq C \frac{h^{r}}{2 k}\left\|\int_{0}^{2 k} \int_{t^{m-4}+t}^{t^{m-2}+t} w_{t t}(\tau) \mathrm{d} \tau \mathrm{d} t\right\|_{r}, \quad m=4, \ldots, N,
\end{aligned}
$$

which yields

$$
\max _{4 \leq m \leq N}\left\|\omega_{w}^{m}-\omega_{w}^{m-2}\right\| \leq C k h^{r}
$$

- Estimation of $\left\|\vartheta_{\Lambda, \delta, h}^{m}-\vartheta_{\Lambda, \delta, h}^{m-2}\right\|$ : For simplicity, we set $z_{\Lambda, \delta, h}^{m}:=\vartheta_{\Lambda, \delta, h}^{m}-\vartheta_{\Lambda, \delta, h}^{m-2}$ for $m=2, \ldots, N$. Then, (2.12) yields

$$
\frac{z_{\Lambda, \delta, h}^{m}-z_{\Lambda, \delta, h}^{m-2}}{2 k}=\frac{\mathrm{i}}{2} \Delta_{h}\left(z_{\Lambda, \delta, h}^{m}+z_{\Lambda, \delta, h}^{m-2}\right)+\delta_{\Lambda, \delta, h}^{m}, \quad m=4, \ldots, N
$$

where

$$
\delta_{\Lambda, \delta, h}^{m}:=-P_{h}\left(\sigma^{m}-\sigma^{m-2}\right)-P_{h}\left(\omega_{w}^{m}-\omega_{w}^{m-2}\right)+\mathrm{i} P_{h}\left(\omega_{\Lambda, \delta}^{m}-\omega_{\Lambda, \delta}^{m-2}\right) .
$$

Taking the $L^{2}$ inner product of $(2.26)$ by $z_{\Lambda, \delta, h}^{m}+z_{\Lambda, \delta, h}^{m-2}$ and then real parts we conclude that

$$
\left\|z_{\Lambda, \delta, h}^{m}\right\|+\left\|z_{\Lambda, \delta, h}^{m-1}\right\| \leq\left\|z_{\Lambda, \delta, h}^{m-1}\right\|+\left\|z_{\Lambda, \delta, h}^{m-2}\right\|+2 k\left\|\delta_{\Lambda, \delta, h}^{m}\right\| \text { for } m=4, \ldots, N \text {. }
$$

By (2.6) and (2.25), we get

$$
\left\|\delta_{\Lambda, \delta, h}^{m}\right\| \leq\left\|\omega_{\Lambda, \delta}^{m}-\omega_{\Lambda, \delta}^{m-2}\right\|+C k\left(k^{2}+h^{r}\right), \quad m=4, \ldots, N .
$$

Let $m \in\{4, \ldots, N\}$. To estimate the difference $\omega_{\Lambda, \delta}^{m}-\omega_{\Lambda, \delta}^{m-2}$ in the $L^{2}$ norm, we introduce the following splitting

$$
\omega_{\Lambda, \delta}^{m}-\omega_{\Lambda, \delta}^{m-2}=\sum_{j=1}^{4} \mathcal{T}_{j}^{m}
$$


where

$$
\begin{aligned}
& \mathcal{T}_{1}^{m}:=\gamma_{\delta}\left(\frac{\Lambda_{\delta, h}^{m}+\Lambda_{\delta, h}^{m-2}}{2}\right)\left[f\left(\left|\gamma_{\delta}\left(\Lambda_{\delta, h}^{m-1}\right)\right|^{2}\right)-f\left(\left|\gamma_{\delta}\left(\Lambda_{\delta, h}^{m-3}\right)\right|^{2}\right)-f\left(\left|w^{m-1}\right|^{2}\right)+f\left(\left|w^{m-3}\right|^{2}\right)\right], \\
& \mathcal{T}_{2}^{m}:=\left[f\left(\left|w^{m-1}\right|^{2}\right)-f\left(\left|w^{m-3}\right|^{2}\right)\right]\left[\gamma_{\delta}\left(\frac{\Lambda_{\delta, h}^{m}+\Lambda_{\delta, h}^{m-2}}{2}\right)-\gamma_{\delta}\left(\frac{w^{m}+w^{m-2}}{2}\right)\right], \\
& \mathcal{T}_{3}^{m}:=f\left(\left|\gamma_{\delta}\left(\Lambda_{\delta, h}^{m-3}\right)\right|^{2}\right)\left[\gamma_{\delta}\left(\frac{\Lambda_{\delta, h}^{m}+\Lambda_{\delta, h}^{m-2}}{2}\right)-\gamma_{\delta}\left(\frac{\Lambda_{\delta, h}^{m-2}+\Lambda_{\delta, h}^{m-4}}{2}\right)-\gamma_{\delta}\left(\frac{w^{m}+w^{m-2}}{2}\right)+\gamma_{\delta}\left(\frac{w^{m-2}+w^{m-4}}{2}\right)\right], \\
& \mathcal{T}_{4}^{m}:=\frac{w^{m}-w^{m-4}}{2}\left[f\left(\left|\gamma_{\delta}\left(\Lambda_{\delta, h}^{m-3}\right)\right|^{2}\right)-f\left(\left|w^{m-3}\right|^{2}\right)\right] .
\end{aligned}
$$

Proceeding as in the proof of Proposition 2.12, we use (2.1), the mean value theorem and (1.5), to obtain

$$
\left\|\mathcal{T}_{2}^{m}\right\| \leq C_{\delta} k\left(h^{r}+\left\|\vartheta_{\Lambda, \delta, h}^{m}\right\|+\left\|\vartheta_{\Lambda, \delta, h}^{m-2}\right\|\right) \text { and }\left\|\mathcal{T}_{4}^{m}\right\| \leq C_{\delta} k\left(h^{r}+\left\|\vartheta_{\Lambda, \delta, h}^{m-3}\right\|\right) .
$$

Combining (2.2), (2.3), the mean value theorem and (1.5), it follows that

$$
\begin{aligned}
& \left\|\mathcal{T}_{3}^{m}\right\| \leq C \sup _{x \in\left[0,8 \delta^{2}\right]}|f(x)|\left[k \sup _{|x| \in[\delta, 2 \delta]}\left|g_{\delta}^{\prime \prime}(x)\right|\left(h^{r}+\left\|\vartheta_{\Lambda, \delta, h}^{m}\right\|+\left\|\vartheta_{\Lambda, \delta, h}^{m-2}\right\|+\left\|\vartheta_{\Lambda, \delta, h}^{m-4}\right\|\right)\right. \\
& \left.+\sup _{|x| \in[0,2 \delta]}\left|g_{\delta}^{\prime}(x)\right|\left(k h^{r}+\left\|z_{\Lambda, \delta, h}^{m}\right\|+\left\|z_{\Lambda, \delta, h}^{m-2}\right\|\right)\right], \\
& \left\|\mathcal{T}_{1}^{m}\right\| \leq C \delta\left[k \sup _{|x| \in\left[0,16 \delta^{2}\right]}\left|f^{\prime \prime}(x)\right|(1+\delta) \sup _{|x| \in[0,2 \delta]}\left|g_{\delta}^{\prime}(x)\right|\left(h^{r}+\left\|\vartheta_{\Lambda, \delta, h}^{m-1}\right\|+\left\|\vartheta_{\Lambda, \delta, h}^{m-3}\right\|\right)\right. \\
& \left.+\sup _{|x| \in\left[0,16 \delta^{2}\right]}\left|f^{\prime}(x)\right|||\left|\gamma_{\delta}\left(\Lambda_{\delta, h}^{m-1}\right)\right|^{2}-\left|\gamma_{\delta}\left(\Lambda_{\delta, h}^{m-3}\right)\right|^{2}-\left|w^{m-1}\right|^{2}+\left|w^{m-3}\right|^{2} \|\right],
\end{aligned}
$$

and

$$
\begin{aligned}
& \left\|\left|\gamma_{\delta}\left(\Lambda_{\delta, h}^{m-1}\right)\right|^{2}-\left|\gamma_{\delta}\left(\Lambda_{\delta, h}^{m-3}\right)\right|^{2}-\left|w^{m-1}\right|^{2}+\left|w^{m-3}\right|^{2}\right\| \\
& \leq C\left[k \sup _{|x| \in[0,2 \delta]}\left|g_{\delta}^{\prime}(x)\right|\left(h^{r}+\left\|\vartheta_{\Lambda, \delta, h}^{m-3}\right\|\right)+\delta\left\|\gamma_{\delta}\left(\Lambda_{\delta, h}^{m-1}\right)-\gamma_{\delta}\left(\Lambda_{\delta, h}^{m-3}\right)-\gamma_{\delta}\left(w^{m-1}\right)+\gamma_{\delta}\left(w^{m-3}\right)\right\|\right] \\
& \leq C_{\delta}\left[k\left(h^{r}+\left\|\vartheta_{\Lambda, \delta, h}^{m-3}\right\|\right)+k \sup _{|x| \in[\delta, 2 \delta]}\left|g^{\prime \prime}(x)\right|\left(h^{r}+\left\|\vartheta_{\Lambda, \delta, h}^{m-1}\right\|+\left\|\vartheta_{\Lambda, \delta, h}^{m-1}\right\|\right)\right. \\
& \left.\quad+\sup _{|x| \in[0,2 \delta]}\left|g^{\prime}(x)\right|\left(k h^{r}+\left\|z_{\Lambda, \delta, h}^{m-1}\right\|\right)\right] .
\end{aligned}
$$

Hence, (2.29), together with (2.30a-d) and (2.11), implies

$$
\left\|\omega_{\Lambda, \delta}^{m}-\omega_{\Lambda, \delta}^{m-2}\right\| \leq \hat{C}_{\delta} k\left(\left\|z_{\Lambda, \delta, h}^{m}\right\|+\left\|z_{\Lambda, \delta, h}^{m-1}\right\|+\left\|z_{\Lambda, \delta, h}^{m-2}\right\|\right)+C_{\delta} k\left(k^{2}+h^{r}\right) .
$$

Now, by (2.27), (2.28) and (2.31) we obtain

$$
\left(1-\hat{C}_{\delta} k\right)\left(\left\|z_{\Lambda, \delta, h}^{m}\right\|+\left\|z_{\Lambda, \delta, h}^{m-1}\right\|\right) \leq\left(1+\hat{C}_{\delta} k\right)\left(\left\|z_{\Lambda, \delta, h}^{m-1}\right\|+\left\|z_{\Lambda, \delta, h}^{m-2}\right\|\right)+C_{\delta} k^{2}\left(k^{2}+h^{r}\right), \quad m=4, \ldots, N,
$$

and by $(2.19 \mathrm{a}-\mathrm{b})$ we conclude that

$$
\begin{aligned}
\left\|z_{\Lambda, \delta, h}^{3}\right\|+\left\|z_{\Lambda, \delta, h}^{2}\right\| & \leq\left\|\vartheta_{\Lambda, \delta, h}^{3}\right\|+\left\|\vartheta_{\Lambda, \delta, h}^{2}\right\|+\left\|\vartheta_{\Lambda, \delta, h}^{1}\right\| \\
& \leq C_{\delta} k\left(k^{2}+h^{r}\right) .
\end{aligned}
$$


Assuming that $\hat{C}_{\delta} k \leq \frac{1}{3}$, and then applying a discrete Grönwall argument on (2.32a-b), we get

$$
\max _{2 \leq m \leq N}\left\|\vartheta_{\Lambda, \delta, h}^{m}-\vartheta_{\Lambda, \delta, h}^{m-2}\right\| \leq C_{\delta} k\left(k^{2}+h^{r}\right)
$$

Finally, using (2.21), (2.11) and (2.24), we have

$$
\left\|\nabla \vartheta_{\Lambda, \delta, h}^{1 / 2}\right\| \leq C_{\delta} \sqrt{k}\left(k+h^{r}\right) \text { and }\left\|\nabla \vartheta_{\Lambda, \delta, h}^{1}\right\| \leq C_{\delta}\left(k^{2}+h^{r}\right)
$$

and (2.20) follows from (2.23), (2.34), (2.33) and (2.24).

We use, now, the estimates of Propositions 2.12 and 2.13 to obtain convergence results for the method (1.8).

Theorem 2.14. Let $d=1$ and $\delta_{0}=\sup _{t \in I_{*}}|w(\cdot, t)|_{\infty}+\sup _{t \in I_{*}}\|w(\cdot, t)\|_{r}+2$. If $W_{h}^{0}=R_{h} w^{0}$ for $h \in(0, \widetilde{h})$, and

$$
\mathcal{C}_{1, \infty} C_{R} h^{r-\frac{1}{2}} \leq 1 \quad \text { and } \mathcal{C}_{1, \infty} \sqrt{C_{A, \delta_{0}} \mathcal{C}_{A, \delta_{0}}}\left(k^{\frac{7}{4}}+h^{r}\right) \leq 1, \quad \forall N \geq \tilde{N}, \quad \forall h \in(0, \widetilde{h})
$$

for some $\tilde{N} \geq \max \left\{t^{*}, \widetilde{N}_{\delta_{0}}\right\}(c f .(2.20))$ and $\widetilde{h} \in(0,1)$, then $(2.10)$ holds. Here, $\mathcal{C}_{1, \infty}, C_{R}, C_{A, \delta_{0}}$ and $\mathcal{C}_{A, \delta_{0}}$ are the constants in (1.3), (1.5), (2.11) and (2.20), respectively.

Proof. Let $h \in(0, \widetilde{h}), N \geq \widetilde{N}$ and $\eta^{\ell}:=R_{h} w^{\ell}-w^{\ell}$ for $\ell=0,1 / 2,1, \ldots, N$. Using (1.3), (1.5), (2.11), (2.20) and (2.35), we have

$$
\begin{aligned}
\left|\Lambda_{\delta_{0}, h}^{m}-w^{m}\right|_{\infty} & \leq\left|\vartheta_{\Lambda, \delta_{0}, h}^{m}\right|_{\infty}+\left|\eta^{m}\right|_{\infty} \\
& \leq \mathcal{C}_{1, \infty}\left(\left\|\vartheta_{\Lambda, \delta_{0}, h}^{m}\right\|^{\frac{1}{2}}\left\|\nabla \vartheta_{\Lambda, \delta_{0}, h}^{m}\right\|^{\frac{1}{2}}+\left\|\eta^{m}\right\|^{\frac{1}{2}}\left\|\nabla \eta^{m}\right\|^{\frac{1}{2}}\right) \\
& \leq \mathcal{C}_{1, \infty}\left[\left(C_{A, \delta_{0}} \mathcal{C}_{A, \delta_{0}}\right)^{\frac{1}{2}}\left(k^{\frac{7}{4}}+h^{r}\right)+C_{R} h^{r-\frac{1}{2}}\left\|w^{m}\right\|_{r}\right] \\
& \leq 1+\sup _{t \in I_{*}}\|w(\cdot, t)\|_{r}, \quad m=0,1 / 2,1, \ldots, N .
\end{aligned}
$$

Thus, we get $\left|\Lambda_{\delta_{0}, h}^{0}\right|_{\infty}<\delta_{0}$ and $\max _{0 \leq m \leq N}\left|\Lambda_{\delta_{0}, h}^{\ell_{m}^{*}}\right|_{\infty}<\delta_{0}$. By Remark 2.7 we conclude that $\Lambda_{\delta_{0}, h}^{\ell_{m}^{*}}=W_{h}^{\ell_{m}^{*}}$ for $m=0, \ldots, N$. Then, combining $(2.11),(2.20)$ and (1.5), the estimates in (2.10) directly follow.

Theorem 2.15. Let $d=2$ or 3 , and $\delta_{0}=\sup _{t \in I_{*}}|w(\cdot, t)|_{\infty}+\sup _{t \in I_{*}}\|w(\cdot, t)\|_{r}+3$. Also, assume that

$$
\begin{array}{rlrl}
|\chi|_{\infty} & \leq C_{\infty} \widetilde{\psi}(h)\|\nabla \chi\|, & & \forall \chi \in S_{h}, \quad \forall h \in(0, \tilde{h}), \\
C_{\infty} C_{R} \widetilde{\psi}(h) h^{r-1} \leq 1, & & \forall h \in(0, \tilde{h}),
\end{array}
$$

and

$$
\inf _{\chi \in S_{h}}\left\{|w(\cdot, t)-\chi|_{\infty}+C_{\infty} \widetilde{\psi}(h)\|\nabla(w(\cdot, t)-\chi)\|\right\} \leq 1, \quad \forall h \in(0, \widetilde{h}), \quad \forall t \in I_{*}
$$

for some $\tilde{h} \in(0,1)$. If $W_{h}^{0}=R_{h} w^{0}$ for $h \in\left(0, \widetilde{h}_{*}\right)$, and

$$
\mathcal{C}_{A, \delta_{0}} C_{\infty} \widetilde{\psi}(h)\left(k^{\frac{3}{2}}+h^{r}\right) \leq 1, \quad \forall N \geq \widetilde{N}, \quad \forall h \in\left(0, \widetilde{h}_{*}\right)
$$

for some $\tilde{N} \geq \max \left\{t^{*}, \widetilde{N}_{\delta_{0}}\right\}(c f .(2.20))$ and $\widetilde{h}_{*} \in(0, \widetilde{h})$, then $(2.10)$ holds. Here, $C_{R}$ and $\mathcal{C}_{A, \delta_{0}}$ are the constants in (1.5) and (2.20), respectively. 
Proof. Let $h \in\left(0, \widetilde{h}_{*}\right), N \geq \widetilde{N}$ and $\eta^{\ell}:=R_{h} w^{\ell}-w^{\ell}$ for $\ell=0,1 / 2,1, \ldots, N$. Using (2.36), for $m=$ $0,1 / 2,1, \ldots, N$, we obtain

$$
\begin{aligned}
\left|\Lambda_{\delta_{0}, h}^{m}-w^{m}\right|_{\infty} \leq & \left|\chi-w^{m}\right|_{\infty}+C_{\infty} \widetilde{\psi}(h)\left\|\nabla\left(w^{m}-\chi\right)\right\| \\
& +C_{\infty} \widetilde{\psi}(h)\left(\left\|\nabla \vartheta_{\Lambda, \delta_{0}, h}^{m}\right\|+\left\|\nabla \eta^{m}\right\|\right), \quad \forall \chi \in S_{h} .
\end{aligned}
$$

From (2.40), (2.38), (2.20), (2.39), (1.5) and (2.37), we conclude that $\left|\Lambda_{\delta_{0}, h}^{0}\right|_{\infty}<\delta_{0}$ and $\max _{0 \leq m \leq N}\left|\Lambda_{\delta_{0}, h}^{\ell_{m}^{*}}\right|_{\infty}<\delta_{0}$. Then, Remark 2.7 implies that $\Lambda_{\delta_{0}, h}^{\ell_{m}^{*}}=W_{h}^{\ell_{m}^{*}}$ for $m=0, \ldots, N$, and we get (2.10) by (2.11), (2.20) and (1.5).

Remark 2.16. Assuming that the finite element spaces $S_{h}$ consist of continuous piecewise polynomial functions on a regular (nondegenerate) partition of $\Omega$, the inverse inequality $(2.36)$ holds with $\widetilde{\psi}(h)=\sqrt{|\ln (\underline{h})|}$ when $d=2$, and $\widetilde{\psi}(h)=\underline{h}^{-1 / 2}$ when $d=3$ (cf. Chap. 5 in [23], Chap. 4.5 in [4]). Here, $\underline{h}$ is the minimum of the diameter of the elements (e.g. triangles) of the partition of $\Omega$ corresponding to $S_{h}$. Also, (2.38) is established by (2.37) and the approximation properties of the interpolant in the $L^{\infty}$ and $H^{1}$ norms (cf. Chap. 4.4 in [4]).

Remark 2.17. Under the assumptions of Theorem 2.14 or Theorem 2.15, we use (2.33) and (1.5) to obtain $\max _{2 \leq m \leq N}\left\|\frac{W_{h}^{m}-W_{h}^{m-2}}{2 k}-w_{t}\left(\cdot, t^{m-1}\right)\right\|=\mathcal{O}\left(k^{2}+h^{r}\right)$ which is an $L^{2}$ approximation estimate for the time derivative of the solution.

Remark 2.18. Taking the $L^{2}$ product of (2.12) by $\vartheta_{\Lambda, \delta, h}^{\ell_{m}^{*}}+\vartheta_{\Lambda, \delta, h}^{j_{m}^{*}}$ and then imaginary parts we obtain $\left\|\nabla \vartheta_{\Lambda, \delta, h}^{1 / 2}\right\|^{2}$ $\leq C\left\|\sigma_{\Lambda, \delta, h}^{0}\right\|\left\|\vartheta_{\Lambda, \delta, h}^{1 / 2}\right\|,\left\|\nabla \vartheta_{\Lambda, \delta, h}^{1}\right\|^{2} \leq C\left\|\sigma_{\Lambda, \delta, h}^{1}\right\|\left\|\vartheta_{\Lambda, \delta, h}^{1}\right\|$ and $\left\|\nabla\left(\vartheta_{\Lambda, \delta, h}^{m}+\vartheta_{\Lambda, \delta, h}^{m-2}\right)\right\|^{2} \leq C\left(\left\|\sigma_{\Lambda, \delta, h}^{m}\right\|+\frac{1}{k} \| \vartheta_{\Lambda, \delta, h}^{m}-\right.$ $\left.\vartheta_{\Lambda, \delta, h}^{m-2} \|\right)\left\|\vartheta_{\Lambda, \delta, h}^{m}+\vartheta_{\Lambda, \delta, h}^{m-2}\right\|$ for $m=2, \ldots, N$. Using (2.11), (2.24) and (2.33), we obtain (2.34) and $\max _{2 \leq m \leq N} \| \nabla\left(\vartheta_{\Lambda, \delta, h}^{m}\right.$ $\left.+\vartheta_{\Lambda, \delta, h}^{m-2}\right) \| \leq C_{\delta}\left(k^{2}+h^{r}\right)$. Thus, under the assumptions of Theorem 2.14 or Theorem 2.15, we arrive at an optimal order "average" $H^{1}$ estimate, i.e., $\max _{2 \leq m \leq N}\left\|\nabla\left(\frac{W_{h}^{m}+W_{h}^{m-2}}{2}-w^{m-1}\right)\right\|=\mathcal{O}\left(k^{2}+h^{r-1}\right)$, which is similar to the one obtained in [24] for the nonlinear Crank-Nicolson method. The $H^{1}$ estimate in (2.10) is stronger and based on a different stability argument.

\subsection{A special case}

Assuming that $d=2$ and the function $f$ has the property $(\mathcal{D})$, we are going to prove the following estimates

$$
\max _{0 \leq m \leq N}\left\|W_{h}^{m}-w^{m}\right\| \leq \mathcal{C}_{B}\left(k^{2}+h^{r}\right) \text { and } \max _{0 \leq m \leq N}\left\|\nabla\left(W_{h}^{m}-w^{m}\right)\right\| \leq \mathcal{C}_{B}\left(k^{\frac{3}{2}}+h^{r-1}+k^{-\frac{1}{2}} h^{r}\right)
$$

for $h \in\left(0, h_{0}\right)$ and $N \geq N_{0}$, where $h_{0} \in(0,1), N_{0} \in \mathbb{N}$, and $\mathcal{C}_{B}$ is a constant independent of $h$ and $N$.

The goal, here, is to get optimal order $L^{2}$ convergence of the method (1.8) avoiding the use of extra assumptions on the finite element spaces to control $L^{\infty}$ norms (cf. (2.36), (2.37) and (2.38) in Theorem 2.15). To do this we will work with the modified scheme $(\Upsilon)$ taking $\nu=\|\nabla \cdot\|$ and estimate, in $L^{2}$ and $H^{1}$ norms, the differences $\vartheta_{\Upsilon, \delta, h}^{1 / 2}:=\Upsilon_{\delta, h}^{1 / 2}-R_{h} w^{1 / 2}$ and $\vartheta_{\Upsilon, \delta, h}^{n}:=\Upsilon_{\delta, h}^{n}-R_{h} w^{n}, n=0, \ldots, N$, where, due to (2.8a), we have $\vartheta_{\Upsilon, \delta, h}^{0}=0$.

Proposition 2.19. Let $\nu=\|\nabla \cdot\|$ and $\delta>\sup _{t \in I_{*}}\|\nabla w(\cdot, t)\|$. If $d=2$ and $f$ satisfies $(\mathcal{D})$, then there exists $M_{\delta} \in \mathbb{N}$ such that

$$
\left\|\vartheta_{\Upsilon, \delta, h}^{1 / 2}\right\| \leq C_{B, \delta}\left(k^{2}+k h^{r}\right) \quad \text { and } \max _{1 \leq m \leq N}\left\|\vartheta_{\Upsilon, \delta, h}^{m}\right\| \leq C_{B, \delta}\left(k^{2}+h^{r}\right), \quad \forall h \in(0,1), \quad \forall N \geq M_{\delta} .
$$

The constant $C_{B, \delta}$ is independent of $h$ and $N$, but depends on $\delta$, the solution or its derivatives, and the data. 
Proof. Let $h \in(0,1)$. We proceed as in the proof of Proposition 2.12, to obtain

$$
\left\|\vartheta_{\Upsilon, \delta, h}^{1 / 2}\right\| \leq C k\left(k+h^{r}+\left\|\omega_{\Upsilon, \delta}^{0}\right\|\right), \quad\left\|\vartheta_{\Upsilon, \delta, h}^{1}\right\| \leq C k\left(k^{2}+h^{r}+\left\|\omega_{\Upsilon, \delta}^{1}\right\|\right)
$$

and

$$
\left\|\vartheta_{\Upsilon, \delta, h}^{m}\right\|-\left\|\vartheta_{\Upsilon, \delta, h}^{m-2}\right\| \leq C k\left(k^{2}+h^{r}+\left\|\omega_{\Upsilon, \delta}^{m}\right\|\right) \text { for } m=2, \ldots, N
$$

with

$$
\omega_{\Upsilon, \delta}^{m}:=\frac{1}{2}\left[F\left(g_{\nu, \delta}\left(t^{i_{m}^{*}} ; \Upsilon_{\delta, h}^{i_{m}^{*}}\right), g_{\nu, \delta}\left(t^{\ell_{m}^{*}} ; \Upsilon_{\delta, h}^{\ell_{m}^{*}}\right)+g_{\nu, \delta}\left(t_{m}^{j_{m}^{*}} ; \Upsilon_{\delta, h}^{j_{m}^{*}}\right)\right)-F\left(w^{i_{m}^{*}}, w^{\ell_{m}^{*}}+w^{j_{m}^{*}}\right)\right]
$$

Let $m \in\{0, \ldots, N\}$. By (2.4) and Lemma 2.10, it follows that

$$
\begin{gathered}
\left\|\omega_{\Upsilon, \delta}^{m}\right\| \leq C_{\delta}\left[\xi_{\delta}\left(\left\|\nabla\left(\Upsilon_{\delta, h}^{\ell_{m}^{*}}-w^{\ell_{m}^{*}}\right)\right\|\right)\left\|\Upsilon_{\delta, h}^{\ell_{m}^{*}}-w^{\ell_{m}^{*}}\right\|+\xi_{\delta}\left(\left\|\nabla\left(\Upsilon_{\delta, h}^{i_{m}^{*}}-w^{i_{m}^{*}}\right)\right\|\right)\left\|\Upsilon_{\delta, h}^{i_{m}^{*}}-w^{i_{m}^{*}}\right\|\right. \\
\left.+\xi_{\delta}\left(\left\|\nabla\left(\Upsilon_{\delta, h}^{j_{m}^{*}}-w^{j_{m}^{*}}\right)\right\|\right)\left\|\Upsilon_{\delta, h}^{j_{m}^{*}}-w^{j_{m}^{*}}\right\|\right]
\end{gathered}
$$

which, together with (1.5), yields

$$
\left\|\omega_{\Upsilon, \delta}^{m}\right\| \leq C_{\delta}\left(h^{r}+\left\|\vartheta_{\Upsilon, \delta, h}^{\ell_{m}^{*}}\right\|+\left\|\vartheta_{\Upsilon, \delta, h}^{i_{m}^{*}}\right\|+\left\|\vartheta_{\Upsilon, \delta, h}^{j_{m}^{*}}\right\|\right)
$$

Substituting (2.45) in (2.43a-b), we get

$$
\begin{aligned}
\left\|\vartheta_{\Upsilon, \delta, h}^{1 / 2}\right\|+\left\|\vartheta_{\Upsilon, \delta, h}^{1}\right\| & \leq \underline{C}_{\delta}\left(k^{2}+k h^{r}\right) \\
\left(1-\underline{C}_{\delta} k\right)\left(\left\|\vartheta_{\Upsilon, \delta, h}^{m}\right\|+\left\|\vartheta_{\Upsilon, \delta, h}^{m-1}\right\|\right) & \leq\left(1+\underline{C}_{\delta} k\right)\left(\left\|\vartheta_{\Upsilon, \delta, h}^{m-1}\right\|+\left\|\vartheta_{\Upsilon, \delta, h}^{m-2}\right\|\right)+C_{\delta} k\left(k^{2}+h^{r}\right), \quad m=2, \ldots, N .
\end{aligned}
$$

Finally, taking $\underline{C}_{\delta} k \leq \frac{1}{3},(2.42)$ follows by a discrete Grönwall argument.

Proposition 2.20. Let $\nu=\|\nabla \cdot\|$ and $\delta>\sup _{t \in I_{*}}\|\nabla w(\cdot, t)\|$. If $d=2$ and $f$ satisfies $(\mathcal{D})$, then there exists an integer $\widetilde{M}_{\delta} \geq M_{\delta}$ such that

$$
\left\|\nabla \vartheta_{\Upsilon, \delta, h}^{1 / 2}\right\| \leq \mathcal{C}_{B, \delta}\left(k^{\frac{3}{2}}+k^{\frac{1}{2}} h^{r}\right) \quad \text { and } \max _{1 \leq m \leq N}\left\|\nabla \vartheta_{\Upsilon, \delta, h}^{m}\right\| \leq \mathcal{C}_{B, \delta} k^{-\frac{1}{2}}\left(k^{2}+h^{r}\right), \quad \forall h \in(0,1), \quad \forall N \geq \widetilde{M}_{\delta}
$$

The constant $\mathcal{C}_{B, \delta}$ is independent of $h$ and $N$, but depends on $\delta$, the solution or its derivatives, and the data.

Proof. Let $h \in(0,1)$ and $N>M_{\delta}$ (cf. Prop. 2.19). For $m=0, \ldots, N$, we define $\omega_{\Upsilon, \delta}^{m}$ as in (2.44). Now, proceeding along the lines of the proof of Proposition 2.13 ( $c f .(2.21)$ and (2.22)), we conclude that

$$
\left\|\nabla \vartheta_{\Upsilon, \delta, h}^{1 / 2}\right\|^{2} \leq C\left(k+h^{r}+\left\|\omega_{\Upsilon, \delta}^{0}\right\|\right)\left\|\vartheta_{\Upsilon, \delta, h}^{1 / 2}\right\|, \quad\left\|\nabla \vartheta_{\Upsilon, \delta, h}^{1}\right\|^{2} \leq C\left(k^{2}+h^{r}+\left\|\omega_{\Upsilon, \delta}^{1}\right\|\right)\left\|\vartheta_{\Upsilon, \delta, h}^{1}\right\|
$$

and

$$
\max _{2 \leq m \leq N}\left\|\nabla \vartheta_{\Upsilon, \delta, h}^{m}\right\|^{2} \leq\left\|\nabla \vartheta_{\Upsilon, \delta, h}^{1}\right\|^{2}+C k^{-1}\left(k^{2}+h^{r}+\max _{2 \leq \ell \leq N}\left\|\omega_{\Upsilon, \delta}^{\ell}\right\|\right) \max _{1 \leq m \leq N}\left\|\vartheta_{\Upsilon, \delta, h}^{m}\right\|
$$

Also, from (2.45) and (2.42), we obtain

$$
\max _{0 \leq m \leq N}\left\|\omega_{\Upsilon, \delta}^{m}\right\| \leq C_{\delta}\left(k^{2}+h^{r}\right)
$$

Substituting (2.48) and (2.42) in (2.47a-b), (2.46) follows. 
Closing the section, we will use Propositions 2.19 and 2.20 to obtain the estimates in (2.41). For that result we do not need any inverse inequality between norms on the finite element spaces.

Theorem 2.21. Let $d=2, \nu=\|\nabla \cdot\|$ and $\delta_{0}=\sup _{t \in I_{*}}\|\nabla w(\cdot, t)\|+3$, and assume that $f$ satisfies $(\mathcal{D})$. If $W_{h}^{0}=R_{h} w^{0}$ for $h \in(0, \widetilde{h})$, and

$$
\mathcal{C}_{B, \delta_{0}}\left(k^{2}+h^{r}\right) \leq \sqrt{k}, \quad \forall N \geq \widetilde{N}, \quad \forall h \in(0, \widetilde{h}),
$$

for some $\widetilde{N} \geq \max \left\{t^{*}, \widetilde{M}_{\delta_{0}}\right\}$ (cf. (2.46)) and $\widetilde{h} \in(0,1)$, then (2.41) holds. Here, $\mathcal{C}_{B, \delta_{0}}$ is the constant in (2.46). Proof. For $h \in(0, \widetilde{h})$ and $N \geq \widetilde{N},(2.49),(1.6)$ and (2.46) imply $\left\|\nabla \Upsilon_{\delta_{0}, h}^{0}\right\|<\delta_{0}$ and $\max _{0 \leq m \leq N}\left\|\nabla \Upsilon_{\delta_{0}, h}^{\ell_{m}^{*}}\right\|<\delta_{0}$. Hence, by Remark 2.9 we have $\Upsilon_{\delta_{0}, h}^{\ell_{m}^{*}}=W_{h}^{\ell_{m}^{*}}$ for $m=0, \ldots, N$, and (2.41) follows from (2.42), (2.46) and (1.5).

Remark 2.22. Let $d=2$. In the case of the cubic Schrödinger equation, i.e. $f(x)=\lambda x$ and $\lambda \in \mathbb{R}$, it is easily seen that $\widetilde{H}\left(W_{h}^{m}, W_{h}^{m-1}\right)=\widetilde{H}\left(W_{h}^{1}, W_{h}^{0}\right)$ for $m=0, \ldots, N$, and $\widetilde{H}\left(W_{h}^{1 / 2}, W_{h}^{0}\right)=\widetilde{H}\left(W_{h}^{1}, W_{h}^{1 / 2}\right)=\widetilde{H}\left(W_{h}^{0}, W_{h}^{0}\right)$, where $\widetilde{H}\left(v_{1}, v_{2}\right):=\frac{\left\|\nabla v_{1}\right\|+\left\|\nabla v_{2}\right\|}{2}-\frac{\lambda}{2}\left(\left|v_{1}\right|^{2},\left|v_{2}\right|^{2}\right)$ for $v_{1}, v_{2} \in H_{0}^{1}(\Omega)$ (cf. also [9]). Then, choosing $W_{h}^{0}=R_{h} w^{0}$ and using the Gagliardo-Nirenberg inequality $|v|_{4}^{4} \leq \mathcal{C}_{G N}\|v\|^{2}\|\nabla v\|^{2}, \forall v \in H_{0}^{1}(\Omega)$, we see that there exists a constant $\widetilde{\delta}>0$, independent of $h$ and $N$, such that $\left\|\nabla W_{h}^{\ell_{m}^{*}}\right\| \leq \widetilde{\delta}$ for $m=0, \ldots, N$, when (i) $\lambda \leq 0$, or (ii) $\lambda>0$ with $\left\|w^{0}\right\|<\left(\frac{2}{\lambda \mathcal{C}_{G N}}\right)^{\frac{1}{2}}$. In these cases, for $\delta>\widetilde{\delta}+\sup _{t \in I_{*}}\|\nabla w(\cdot, t)\|$ and $\nu=\|\nabla \cdot\|$, the method (1.8) is written in the form (2.8), and (2.41) is obtained from (1.5) and the estimates of Propositions 2.19 and 2.20. In this way, (2.49) is avoided.

\section{REFERENCES}

[1] S.A. Akhamanov, A.P. Sukhonorov and R.V. Khoklov, Self-focusing and self-trapping of intense light beams in a nonlinear medium. Sov. Phys. JETP 23 (1966) 1025-1033.

[2] G.D. Akrivis, Finite difference discretization of the cubic Schrödinger equation. IMA J. Numer. Anal. 13 (1993) 115-124.

[3] G.D. Akrivis, V.A. Dougalis and O.A. Karakashian, On fully discrete Galerkin methods of second-order temporal accuracy for the nonlinear Schrödinger equation. Numer. Math. 59 (1991) 31-53.

[4] S.C. Brenner and L.R. Scott, The mathematical theory of finite element methods. Texts Appl. Math. 15, Springer-Verlag, New York (1994).

[5] H. Brezis and T. Gallouet, Nonlinear Schrödinger evolution equations. Nonlinear Analysis 4 (1980) 677-681.

[6] T. Cazenave and A. Haraux, Introduction aux problémes d'évolution semi-linéaires. Ellipses, Paris (1990).

[7] R.Y. Chiao, E. Garmire and C. Townes, Self-trapping of optical beams. Phys. Rev. Lett. 13 (1964) 479-482.

[8] A. Cloot, B.M. Herbst and J.A.C. Weideman, A numerical study of the nonlinear Schrödinger equation involving quintic terms. J. Comput. Phys. 86 (1990) 127-146.

[9] Z. Fei, V.M. Pérez-García and L. Vázquez, Numerical simulation of nonlinear Schrödinger systems: a new conservative scheme. Appl. Math. Comput. 71 (1995) 165-177.

[10] Y. Jingqi, Time decay of the solutions to a nonlinear Schrödinger equation in an exterior domain in $\mathbb{R}^{2}$. Nonlinear Analysis 19 (1992) 563-571.

[11] O. Karakashian, G.D. Akrivis and V.A. Dougalis, On optimal order error estimates for the nonlinear Schrödinger equation. SIAM J. Numer. Anal. 30 (1993) 377-400.

[12] O. Karakashian and Ch. Makridakis, A space-time finite element method for the nonlinear Schrödinger equation: The discontinuous Galerkin method. Math. Comp. 67 (1998) 479-499.

[13] H.Y. Lee, Fully discrete methods for the nonlinear Schrödinger equation. Comput. Math. Appl. 28 (1994) 9-24.

[14] H.A. Levine, The role of critical exponents in blowup theorems. SIAM Review 32 (1990) 262-288.

[15] H. Nawa, Asymptotic profiles of blow-up solutions of the nonlinear Schrödinger equation with critical power nonlinearity. $J$. Math. Soc. Japan 46 (1994) 557-586.

[16] A.C. Newell, Solitons in mathematics and mathematical physics. CBMS Appl. Math. Ser. 48, SIAM, Philadelphia (1988).

[17] J.J. Rasmussen and K. Rypdal, Blow-up in nonlinear Schroedinger equations-I: A general review. Physica Scripta 33 (1986) 481-497. 
[18] M.P. Robinson and G. Fairweather, Orthogonal spline collocation methods for Schrödinger-type equations in one space variable. Numer. Math. 68 (1994) 355-376.

[19] K. Rypdal and J.J. Rasmussen, Blow-up in nonlinear Schroedinger equations-II: Similarity structure of the blow-up singularity. Physica Scripta 33 (1986) 498-504.

[20] J.M. Sanz-Serna, Methods for the numerical solution of the nonlinear Schroedinger equation. Math. Comp. 43 (1984) $21-27$.

[21] W.A. Strauss, Nonlinear wave equations. CBMS Regional Conference Series Math. No. 73, AMS, Providence, RI (1989).

[22] V.I. Talanov, Self-focusing of wave beams in nonlinear media. JETP Lett. 2 (1965) 138-141.

[23] V. Thomée, Galerkin finite-element methods for parabolic problems. Springer Series Comput. Math. 25, Springer-Verlag, Berlin, Heidelberg (1997).

[24] Y. Tourigny, Optimal $\mathrm{H}^{1}$ estimates for two time-discrete Galerkin approximations of a nonlinear Schrödinger equation. IMA J. Numer. Anal. 11 (1991) 509-523.

[25] M. Tsutsumi and N. Hayashi, Classical solutions of nonlinear Schrödinger equations in higher dimensions. Math. Z. 177 (1981) 217-234.

[26] V.E. Zakharov, Collapse of Langmuir waves. Sov. Phys. JETP 35 (1972) 908-922.

To access this journal online: www.edpsciences.org 